\title{
Index rerum ad Vol. 157
}

\section{Confecit: C. Loeb-Schürch}

$(\mathrm{B}) \approx$ Buchbesp $\Gamma$ echungen - Book reviews - Livres nouveaux $(\mathrm{V})=$ Vortrag - Report Communication

Absolute glaucoma; histological changes of the trabecular mesh-work in the eyes with absolute glaucoma, 306

Aderhaut, v. Aderhautmelanom, Leukoenzephalitis, Zyklitis

Aderhautmelanom; licht- und elek-tronenmikroskopische Beobach-tungen an Gefäßeinbrüchen bei Aderhautmelanom, 414

Adjuvantien, v. Cortisonglaukom

Affe, v. Cortisonglaukom

After-image, v. Nachbildprüfung

Ageing, v. Trabecular meshwork

Albinismus, v. Retinal damage

Allergie, v. Leukoenzephalitis

Allergie und Auge, 398 (B)

Altersveränderungen, v. Trabecular meshwork

Amblyopie, v. Optomotorische Reize

Amotio retinae, v. Myopie und Amotio

Amsler, Marc, 401

Anatomie, v. Musculus rectus, Ophthalmic artery, Trabecular meshwork

Angiographie, v. Angiographie fluorescente, Ophthalmic artery, Retinal circulation times,

Zyklitis

Angiographie fluorescente; Гangio-graphie fluorescente dans Гembolie et la thrombose des vaisseaux rétiniens, 407

Angle iridocornéen, v. Absolute glaucoma, Trabecular meshwork

Anterior chamber, v. Vorderkammer-linsen

Apparat, v. Nachbildprüfung, Stereoskop

Aqueous humor, v. Chlorpromazine

Arterial tension, v. Ophthalmo-dynamographische Befunde

Augenbinnendruck, v. Chlorpromazine, Cortisonglaukom, Dislokation der Linse, Hypotension oculaire, Moses-Effekt, Ophthalmodynamo-graphische Befunde

Augeninnenmuskeln, v. Cortisonglaukom

Augenkomplikationen, v. Dislokation der Linse, Phlegmon de Torbite, Retinal damage,

Retinoblastoma

Augenmuskellähmungen, 83 (B)

Augenmuskeln, v. Electromyography, Vertical effects

Binocularsehen, v. Sekundärdiver-

genz, Stereoskop Biochemie, v. Chlorpromazine,

Lactic dehydrogenases, Phospha- 
tases Biological resection, v. Chondro-

sarcoma Blutdruck, v. Ophthalmodynamo-

graphische Befunde Book reviews, 83 (B), 161 (B),

398 (B) Buchbesprechungen, 83 (B), 161 (B),

398 (B)

Cataract, v. Cryoextractor

Cerebral circulatory disturbance, v. Ophthalmodynamographische Befunde

Chambre antérieure, v. Vorder-kammerlinsen

Champ visuel, v. Ophthalmodynamographische Befunde

Chien, v. Musculus rectus, Optic nerve

Childhood; the eye in childhood, 163 (B)

476

Index rerum ad Vol. 157

Chirurgie, v. Chondrosarcoma, Cryoextractor, Keratectasia, Vertical effects, Vorderkammerlinsen

Chirurgie des maladies vasculaires de la rétine et traitement préventif du décollement rétinien, 162 (B)

Chloride, v. Chlorpromazine

Chlorpromazine; changes of intraocular pressure and of chloride, sodium and potassium

concentration in aqueous humor and serum of rabbits following chlorpromazine, 279

Chondrosarcoma; primary 'mesen-chyma $\mathrm{\Gamma}$ chondrosarcoma of the orbit (Chondrosarcoma mesen-chymale primitivum orbitae) treated by biological resection, 24

Chorioidea, v. Aderhautmelanom, Leukoenzephalitis, Zyklitis

Circulation, v. Angiographie fluores-cente, Ophthalmodynamographi-sche Befunde, Retinal circulation

Complications oculaires, v. Dislo-kation der Linse, Phlegmon orbitaire, Retinal damage, Retino-

blastoma

Congenital glaucoma; the patho-genesis of congenital glaucoma, 88 (B)

Contact lenses; corneal and scleral contact lenses. Proceedings of the International Congress, 166

(B)

Cornea, v. Corneal thinning syndrome, Descemet's membrane, Ex-trakorneale

Hornhauttransplanta-tion, Keratectasia, Moses-Effekt, Walzenförmige Hornhautverkrüm-mung,

Zellkontakte

Corneal graft, v. Extrakorneale

Hornhauttransplantation, Keratectasia

Corneal thinning syndrome: Kerato-leptynsis, 263

Corps ciliaire, v. Phosphatases, Zyklitis

Corps étranger, v. Fremdkörper

Corps vitré, v. Phosphatases, Photo-koagulation, Zyklitis

Correspondance anormale, v. Sekundärdivergenz

Cortisonglaukom; experimentelle Studien zum Cortisonglaukom. 3. Die Wirkung von reinen

Gluco-corticosteroiden und Adjuvantien auf isolierte intraokuläre Muskel-streifen des

Rhesusaffen, 65

Cristallin, v. Cryoextractor, Dislo-kation der Linse, Vorderkammer-linsen

Cryoextractor; experimental study on the behaviour of cryoextractor at various temperatures, 313 
Cryopexia, v. Temperature measurement

Current concepts in ophthalmology, 83 (B)

Cyclite, v. Zyklitis

Décollement rétinien, v. Myopie und Amotio

Décollement rétinien; le décollement rétinien. 4e colloque du Club Jules Gonin, 165 (B)

Dégénérescence pigmentaire, v. Vitiligo

Dégénérescence rétinienne, v. Retinal damage

Déhydrogénase lactique, v. Lactic dehydrogenase

Dermatoglyphische Befunde, v. Oral-Facial-Digital-Syndrom

Dermato-oftalmologia, 163 (B)

Descemet's membrane; electron microscopic observations of the early development of the human corneal endothelium and Descemet's membrane, 451

Development, v. Descemet's membrane, Oral-Facial-Digital-Syndrom, Optic nerve

Diabetes, v. Hypotension oculaire

Diagnostik, v. Electromyography of extraocular muscles, Lactic dehy-drogenases, Orbitopneumotomo-graphy

Differenzierung, retinale, v. Optic nerve

Dislokation der Linse; zur Prognose der traumatischen Dislokation der Linse, 301

Divergence secondaire, v. Sekundärdivergenz

Index rerun! ad Vol. 157

477

Dog, v. Musculus rectus, Optic nerve Duane's syndrome, v. Electro-

myography Durchblutungsstörungen des Auges

und Biopsie der Arteria temporalis,

400 (B) Dyskranio-Dysphalangie, v. Oral-

Facial-Digital-Syndrom Dystrophie marginale, v. Corneal

thinning syndrome

Echo-Encephalographie; klinische Echo-Encephalographie, 164 (B)

Electromyography of extraocular muscles in internuclear conditions, 110

Electroretinography; the clinical value of electroretinography, 88 (B)

Elektronenmikroskopie, v. Aderhaut-melanom, Descemet's membrane, Retinal damage,

Zellkontakte

Elektroretinogramm, v. Optic nerve

Embolie rétinienne, v. Angiographie fluorescente

Embryologie, v. Descemet's membrane, Oral-Facial-Digital-Syndrom, Optic nerve

EMG, v. Electromyography

Entwicklung, v. Descemet's membrane, Oral-Facial-Digital-Syndrom, Optic nerve

Entzündung, v. Phlegmon de Гorbite, Zyklitis

Epithelium coméen, v. Moses-Effekt, Zellkontakte

Epithelium pigmentaire, $v$. Retinal damage

ERG, v. Optic nerve

Excentrische Fixation, v. Optomoto-rische Reize

Excitation optomotrice, v. Optomo-torische Reize

Exophthalmus, v. Orbito-pneumo-tomography 
Experimental research, v. Chlor-promazine, Cortisonglaukom, Cryoextractor, Moses-Effekt, Optic nerve, Phosphatases, Retinal damage, Temperature measurement

Extrakorneale Hornhauttransplan-tation bei nodulärer nekrotisieren-der Skleritis, 374

Fixation excentrique, v. Optomoto-rische Reize

Fluids, intraocular, v. Lactic dehy-drogenases

Fluorescein photography, v. Angiographie fluorescente, Retinal circulation times, Zyklitis

Fluoreszenzangiographie der Retina,

85 (B)Fluoroangiographie, v. Angiographie

fluorescente, Retinal circulation times, Zyklitis

Foreign body, v. Fremdkörper

Fortschritte der Augenheilkunde, 164 (B)

Fremdkörper; Fernseh-Röntgen intraokularer Fremdkörper. Ein neues Verfahren zur

Lokalisation und Extraktion, 398 (B)

Fremdkörper; histologische Befunde bei im menschlichen Auge ver-bliebenen Fremdkörpern, 208

Fundus angiography; atlas of fluorescence fundus angiography, 167 (B)

Fusion, v. Sekundärdivergenz, Stereoskop

Gefäße, v. Angiogiaphie fluorescente,

Ophthalmic artery, Ophthalmo-

dynamographische Befunde, Photo-

koagulation, Retinal circulation

times Genetics, v. Oral-Facial-Digital-

Syndrom Geronto-Ophthalmologie, v. Trabe-

cular meshwork Gesichtsfeld, v. Ophthalmodynamo-

graphische Befunde Glaskörper, v. Phosphatases, Photo-

koagulation, Zyklitis Glaucoma simplex. A long-term

clinical study, 88 (B) Glaucoma surgery; atlas of glaucoma

surgery, 165 (B) Glaucoma. Symposium at Tutzing

castle, 164 (B) Glaucoma Symposium; first South

American Glaucoma Symposium,

$86 \quad$ (B)

Glaukom, v. Absolute glaucoma, Cortisonglaukom, Ophthalmo-dynamographische Befunde, Trabecular meshwork

rum ad Vol. 157

478 Index rei

Glucocorticosteroide, v. Cortison-

glaukom Greffe cornéenne, v. Extrakorneale

HornhauÜГansplaníation, Kerat-

ectasia

Haemorrhagien, v. Thalassaemia

Hautleistensystem, v. Oral-Facial-Digital-Syndrom

Hérédité, v. Oral-Facíal-Digital-Syndrom

Histochemie, v. Phosphatases 
Histologie, v. Absolute glaucoma, Aderhautmelanom, Chondro-sarcoma, Extrakorneale Hornhaut-transplantation, Fremdkörper, Leukoenzephalitis, Musculus rectus, Optic nerve, Retinal damage, Trabecular meshwork, Zellkontakte

Hornhaut, v. Corneal thinning syndrome, Descemet's membrane, Extrakorneale

Hornhauttransplan-tation, Keratectasia, Moses-Effekt, Walzenförmige Hornhautver-krümmung, Zellkontakte

Hornhautepithel, v. Moses-Effekt, Zellkontakte

Humeur aqueuse, v. Chlorpromazine

Hund, v. Musculus rectus, Optic nerve

Hypertonie, v. Ophthalmodynamo-graphische Befunde

Hypotension oculaire; les incidences de Thypotension oculaire sur la myopie des diabétiques, 36 Immunbiologie, v. Leukoenzephalitis

Inflammation, v. Phlegmon de Гorbite, Zyklitis

Inflammations; Studies on causal relations in endogenous, non-purulent, ocular inflammations, observations in a sample of 420 patients with various ocular inflammations, compared with findings in a control group of 200 people, 161 (B)

Intraoculardruck, v. Chlorpromazine, Cortisonglaukom, Dislokation der Linse, Hypotension oculaire, Moses-Effekt, Ophthalmodynamo-graphische Befunde

Intraoculare Flüssigkeit, v. Lactic dehydrogenases

Intraocularer Fremdkörper, v. Fremdkörper

Intraocular muscles, v. Cortisonglaukom

Iridocorneal angle, v. Absolute glaucoma, Trabecular meshwork

Kalium, v. Chlorpromazine Kammerwasser, v. Chlorpromazine Kammerwinkel, v. Absolute glaucoma, Trabecular meshwork Kaninchen, v. Chlorpromazina,

Phosphatases, Temperature

measurement Katarakt, v. Cryoextractor Keratectasia; a case of extreme

keratectasia treated with kerato-

plasty, 274 Keratoconus, v. Corneal thinning

syndrome Keratoleptynsis, v. Corneal thinning

syndrome Keratoplastik, v. Extrakorneale

Hornhauttransplantation, Keratectasia Keratotorus, v. Walzenförmige

Hornhautverkrümmung Komplikationen in der Augen-

chirurgie und ihre Behandlung,

399 (B) Korrespondenz, anomale, v. Sekun-

därdivergenz Kreislauf, v. Angiographie fluo-

rescente, Ophthalmodynamogra-

phische Befunde, Retinal

circulation Kreislaufstörung, zerebrale, v. Oph-

thalmodynamographische Befunde

Lactic dehydrogenase activity in intraocular fluids. Its value as a sign of intraocular tumor, 223

Lähmung, v. Electromyography Lapin, v. Chlorpromazine, Phosphatases, Temperature

measurement LDH, v. Lactic dehydrogenase Lens, v. Cryoextractor, Dislokation

der Linse, Vorderkammerlinsen Lentilles camérulaires, v. Vorderkammerlinsen

Index rerum ad Vol. 157

479 
Leukoenzephalitis; das histopatho-logische Bild der zentralen chorio-retinalen Veränderungen bei sub-akuter sklerosierender Leukoenzephalitis (van Bogaert), 442

Leukoenzephalitis; das klinische Bild der zentralen chorioretinalen Veränderungen bei subakuter sklerosierender Leukoenzephalitis (van Bogaert), 427

Linse, v. Cryoextracíor, Dislokation der Linse, Vorderkammerlinsen

Lipidosis, v. Niemann-Pick lipidosis

Liquides oculaires, v. Lactic dehydrogenases

Livres nouveaux, 83 (B), 161 (B), 398 (B)

Malformations, v. Oral-Facial-Digital-

Syndrom Marginal dystrophy, v. Coraeal

thinning syndrome Melaninprobe, v. Aderhautmelanom Melanom, v, Aderhautmelanom

Microscopie électronique, v. Aderhautmelanom, Descemet's membrane, Retinal damage, Zell-

kontakte Mißbildungen, v. Oral-Facial-Digital-

Syndrom Monkey, v. Cortisonglaukom Moses-Effekt; über den Einfluß des Cornea-Epithels auf den Moses-Effekt, 60 Motilität, v. Vertical effects Muscles intraoculaires, v. Cortisonglaukom

Muscles oculaires, v. Electro-myography, Vertical effects Musculus rectus; Beitrag zur Anatomie des Musculus rectus externus und des Musculus rectus internus bei Hund und Mensch, 381 Myopie, v. Hypotension oculaire Myopie und Amotio retinae. Statisti-

sche Analyse von 800 Fallen, 249 Myotomie, v. Vertical effects

Nachbildprüfung; ein neuer Apparat

zur Nachbildprüfung, 131 Nachruf, v. Amsler, Marc

Natrium, v. Chlorpromazine

Necrologue, v. Amsler, Marc

Netzhaut, v. Angiographie fluores-cente, Leukoenzephalitis, Niemann-Pick lipidosis, Optic

nerve, Photo-koagulation, Retinal circulation times, Retinal damage, Temperature measurement, Vitiligo

Netzhautablösung, v. Myopie und Amotio

Netzhautdegeneration, v. Retinal damage

Neurologie; Einführung in die klinische Neurologie, 83 (B)

Neuro-Ophthalmologie, v. Electro-myography, Leukoenzephalitis

Niemann-Pick lipidosis; retinal functions in Niemann-Pick lipidosis. Ophthalmological aspects of the chronic form of sphingomyelin lipidosis, 349

Obituary, v. Amsler, Marc

Operation, v. Chondrosarcoma, Cryoextractor, Keratectasia, Vertical effects,

Vorderkammerlinsen

Ophthalmic artery anomalies, 321

Ophthalmodynamographische Be-funde beim Glaukom, 1

Ophthaîmodynamometrie, v. Ophthalmodynamographische Befunde

Ophthalmological Societies of the United Kingdom; Transactions of the Ophthalmological

Societies of the United Kingdom, 84 (B)

Ophthalmological Society of New Zealand; Transactions of the Ophthalmological Society of

New Zealand, 161 (B)

Ophthalmology, 87 (B)

Ophthalmoplegie, v. Electro-myography

Optic nerve; effect of section of the optic nerve on histological differentiation and electrical activity of the retina in dogs. A study of centrifugal influences on the ERG, 293 
Optica fisiológica clínica, 400 (B)

Opticus, v. Optic nerve

Optique médicale pratique, 399 (B)

480

Index rerum ad Vol. 157

Optokinetic stimulation, v. Opto-motorische Reize

Optomotorische Reize; Wirkung optomotorischer Reize auf Fixa-tionsort und

Auflösungsvermögen amblyoper Augen mit exzentri-scher Fixation, 135

Oral-Facial-Digital-Syndrom; ein klinisch-genetischer und dermato-glyphischer Beitrag zur

Nosologie des Oral-Facial-Digital-Syndroms, 178

Orbita, v. Chondrosarcoma, Orbito-pneumotomography, Phlegmon de Гorbite

Orbitalphlegmone, v. Phlegmon de Гorbite

Orbito-pneumotomography; experience on orbito-pneumotomography, 462

Orthoptik, v. Stereoskop

Ottica fisiopatologica; compendio di ottica fisiopatologica, 86 (B)

Paediatro-Ophthalmologie, v. Nie-

mann-Pick lipidosis, Zyklitis Palsy, v. Eleclromyography Parese, v. Electromyography Pars planitis, v. Zyklitis Phlegmon de Torbite d'origine

dentaire, 14 Phosphatases in ocular tissues. Ciliary epithelium, mast cells of ciliary processes and vitreous cells, 231 Photic damage, v. Retinal damage Photokoagulation; Behandlung der proliferativen Veränderungen des Augenhintergrundes mit Photokoagulation, 154

Pigmentdegeneration, v. Vitiligo Pigmentepithel, v. Retinal damage Pneumotomography, v.

Orbito-pneumotomography Post-image, v. Nachbildprüfung Potassium, v. Chlorpromazine

Pression oculaire, v. Chlorpromazine, Cortisonglaukom, Dislokation der Linse, Hypotension oculaire, Moses-Effekt, Ophthalmodynamo-graphische Befunde Problèmes actuels en

ophtalmologie, 85 (B)

Proliferation, retinale, v. Photokoagulation

Pulpitis gangraenosa, v. Phlegmon de Torbite

Rabbit, v. Chlorpromazine, Phosphatases, Temperature measurement

Radiation, v. Retinoblastoma

Radiology, v. Ophthalmic artery, Orbitopneumotomography

Rat, v. Retinal damage

Ratte, v. Retinal damage

Recherches expérimentales, v. Chlorpromazine, Cortisonglaukom, Cryo-extractor, Moses-Effekt,

Optic nerve, Phosphatases, Retinal damage, Temperature measurement

Refraktion, v. Hypotension oculaire, Myopie und Amotio, Retincscopy

Refrazione; i vizi di refrazione, 166 (B)

Réseau trabéculaire, v. Absolute glaucoma, Trabecular meshwork

Retina, v. Angiographie fluorescente, Leukoenzephalitis, Niemann-Pick lipidosis, Optic nerve,

Photokoagulation, Retinal circulation times, Retinal damage, Temperature measurements,

Vitiligo

Retinal circulation times. Studies by means of fluorescein rapid sequence photography, 89

Retinal damage by visible light in albino rats. An electron microscope study, 43

Retinal detachment, v. Myopie und Amotio

Retinal detachment; atlas of diagnostic techniques and treatment of retinal detachment, 165 (B) 
Retinal detachment; vitreoretinal pathology and surgery in retinal detachment, 84 (B)

Retinal differentiation, v. Optic nerve

Retinoblastoma; facial changes due to radiation in cases of retinoblastoma, 268

Retinopathia diabetica; Atlas der Retinopathia diabetica, 161 (B)

Rétinopathie pigmentaire, v. Vitiligo

Index rerum ad Vol. 157

481

Retinoscopy; a simplified explanation

of retinoscopy, 104 Retraction syndrome, v. Electro-

myography Rhesusaffe, v. Cortisonglaukom

Sauna; die Einwirkung der Sauna bei Augenerkrankungen, insbeson-dere beim Grünen Star (Glaukom) , 161 (B)

Schielen, v. Optomotorische Reize, Schielkinder, Sekundärdivergenz, Stereoskop, Vertical effects

Schielkinder; neue Prinzipien in der Behandlung von Va- bis 3jährigen Schielkindern, 285

Sehen und Verkehr, 162 (B)

Sekundärdivergenz; Behandlung der anomalen Korrespondenz durch artifizielle

Sekundärdivergenz, 142

Sensory inhibition, 163 (B)

Singe, v. Cortisonglaukom

Sinus maxillaris, v. Orbito-pneumo-tomography, Phlegmon de Гorbite

Skiaskopie, v. Retinoscopy

Skleritis, v. Extrakorneale Hornhaut-transplantation

Sodium, v. Chlorpromazine

Squint, v. Optomotorische Reize, Schielkinder, Sekundärdivergenz, Stereoskop, Vertical effects

Stereoskop; Übungen an einem neuartigen Stereoskop, 201

Strabismus, v. Optomotorische Reize, Schielkinder, Sekundärdivergenz, Stereoskop, Vertical

effects

Strabismus in childhood, 400 (B)

Strabismus concomitans; Unter-suchungen über die Heredität des Strabismus concomitans, 163

(B)

Surgery, v. Chondrosarcoma, Cryo-extractor, Keratectasia, Vertical effects, Vorderkammerlínsen

Surgery; principles of head and neck surgery, 165 (B)

Syndrome, v. Corneal thinning syndrome, Electromyography, Leukoenzephalitis, Niemann-Pick lipidosis, Oral-Facial-Digital-Syndrom

Tastleistensystem, v. Oral-Facial-Digital-Syndrom

Temperature measurement in the retina by the method of 'electronic switch' during cryopexia, 391

Tension artérielle, v. Ophthalmo-dynamographische Befunde

Tension oculaire, v. Chlorpromazine, Cortisonglaukom, Dislokation der Linse, Hypotension oculaire, Moses-Effekt, Ophthalmodynamo-graphische Befunde

Thalassaemia; high incidence of thalassaemia in patients with intraocular haemorrhages, 343

Therapíe, v. Chondrosarcoma, Extrakorneale Hornhauttransplantation, Keratectasia, Optomotorische Reize, Photokoagulation, Retino-blastoma, Schielkinder, Sekundärdivergenz, Vorderkammerlinsen, Zyklitis 
Thrombose rétinienne, v. Angio-graphie fluorescente

Tonometrie, v. Moses-Effekt

Trabecular meshwork, v. Absolute glaucoma

Trabecular meshwork; hislological changes of the trabecular meshwork in the healthy eye depending on age, 119

Trachome; sur le problème du trachome, 398 (B)

Transscleral cryopexia, $v$. Temperature measurement

Trauma, v. Dislokation der Linse, Fremdkörper

Tumeurs; les tumeurs de l'æil et de ses annexes, 83 (B)

Tumor, v. Aderhautmelanom,

Chondrosarcoma, Lactic dehydro-genase, Retinoblastoma

Ultrasonics in ophthalmology,

399 (B) Untersuchungsmethodik, v. Nach-

bildprüfung, Orbito-pneumotomo-

graphy, Retinal circulation times,

Retinoscopy Uveitis, v. Zyklitis

Vaisseaux, v. Angiographie fluorescente, Ophthalmic artery, Oph-thalmodynamographische

Befunde, Photokoagulation, Retinal circulation times

482

Index Terum ad Vol. 157

Varia, 168, 248

Vererbung, v. Oral-Facial-Digital-Synd $\Gamma$ om

Vertical effects of horizontal $\Gamma$ ecti myotomy, 193

Vessels, v. Angiographie fluorescente, Ophthalmic artery, Ophthalmo-dynamographische

Befunde, Photo-koagulation, Retinal circulation times

Vieillissement, v. Trabecular mesh-work

Vision binoculaire, v. Sekundär-divergenz, Stereoskop

Visual field, v. Ophthalmodynamo-graphische Befunde

Vitiligo; unilateral sectorial pigmentary degeneration and vitiligo, 357

Vitreous body, v. Phosphatases, Photokoagulation, Zyklitis

Vorderkammer, v. Vorderkammer-linsen

Vorderkammerlinsen; Ergebnisse nach Einpflanzung von Vorderkammerlinsen, 76

Walzenförmige Hornhautverkrüm-mung; über die walzenförmige Vorwölbung und Verdünnung der unteren Hornhauthälfte, 328

Zellkontakte; die funktionelle Be-deutung der Zellkontakte im Horn-hautepithel, 362

Zentralarterien-Verschluß, v. Angiographie fluorescente

Zentralvenen-Thrombose, v. Angiographie fluorescente

Ziliarkörper, v. Phosphatases, Zyklitis

Zyklitis; chronische Zyklitis im Kindesalter, 169

Index autorum ad Vol. 157

Confecit: C. Loeb-Schürch

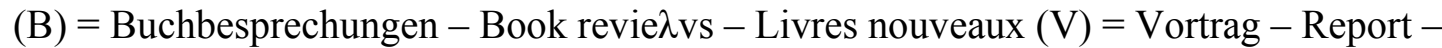

Communication

Alessio, L., 343 Amemiya, T., 462

Becker, 83 (B) 
Békésy, G. von, v. Von Békésy, G.

Bertoni, G., 343

Betlelheim, H., 1

Blagojevic, M., 14

Blumenthal, M., 274

Caldeira, J. A. F., 279 Casanovas, J., 163 (B) Castellazzo, R., 43

Charamis, J., 407 Cibis, P. A., 84 (B) Contino, F., 86 (B) Coscas, G, 399 (B) Csapody, I., 131

Dake, C. L., 88 (B)

De Decker, W., 142

Dieckhues, B., 398 (B)

Dolenek, 161 (B)

Drews, 83 (B)

D. Dufour, 162 (B), 165 (B)

Dymitrowska, Maria, 119, 306 